\title{
The impact of transit monetary costs on transport inequality
}

\author{
Daniel Herszenhut* \\ Universidade Federal do Rio de Janeiro, Brazil \\ Institute for Applied Economic Research - IPEA, Brazil \\ Rafael H. M. Pereira \\ Institute for Applied Economic Research - IPEA, Brazil \\ Licinio da Silva Portugal \\ Universidade Federal do Rio de Janeiro, Brazil \\ Matheus Henrique de Sousa Oliveira \\ Universidade Federal do Rio de Janeiro, Brazil
}

\section{Declarations of interest}

None

\section{Highlights}

- The study examines how incorporating monetary costs into accessibility measures may impact transport inequality assessment.

- Disregarding monetary constraints do overestimate accessibility levels.

- Stricter monetary constraints, however, do not necessarily lead to more unequal scenarios.

- How inequality assessments are affected depend on the combinations of time and monetary costs considered.

- Including monetary constraints on accessibility estimates may help diagnosing transport inequalities.

\begin{abstract}
Transport inequality analyses are often informed by accessibility estimates based solely on travel time impedance, ignoring other elements that might hinder access to activities, such as the monetary cost of a trip. This paper examines how and to what extent simultaneously incorporating both time and monetary costs into accessibility measures may impact transport inequality assessment. We calculate job accessibility by transit in the city of Rio de Janeiro, Brazil, using cumulative opportunity measures under distinct combinations of temporal and monetary thresholds, and compare how inequality levels vary across different scenarios. We find that the most common research practice of disregarding monetary costs tends to overestimate accessibility levels. However, stricter monetary constraints do not necessarily result in more unequal scenarios. How accessibility inequality is affected by monetary costs is highly dependent on what
\end{abstract}

* Corresponding author. E-mail: daniel.santos@pet.coppe.ufrj.br. Postal address: Av. Horácio Macedo, 2030. Bloco H - Sala 106 - Ilha do Fundão. 21941-914 - Rio de Janeiro 
combinations of temporal and monetary cut-offs are considered in the analysis. In the case of Rio, opting for lower monetary thresholds when looking at shorter trips leads to inequality levels lower than those found in the no monetary threshold scenario, but results in higher inequality levels when allowing for longer trips. We find that the impact of monetary costs on transport inequality estimates depend on a complex interaction between fare policies, the spatial organization and operational characteristics of transit systems, and the spatial co-distribution of opportunities and residential locations. The paper thus highlights that conclusions and policy recommendations derived from transport inequality analyses can be affected in non-intuitive ways by the interplay between temporal and monetary constraints. Future research should investigate how different combinations of travel time and monetary costs thresholds affect inequality estimates in different contexts.

\section{Keywords}

Accessibility; Socio-spatial inequalities; Equity; Monetary costs; Fare policy; Rio de Janeiro

\section{Introduction}

Accessibility has been increasingly considered as a key transport policy goal in recent years. The understanding that higher accessibility levels indicates higher potential for personal fulfillment and satisfaction (Martens, 2012) and that transport policies should consider minimum accessibility standards for key destinations and reduce inequalities of opportunities (Pereira et al., 2017) leads researchers and decisionmakers to investigate how such policies affect different regions and social groups (Lucas et al., 2016). Most studies measure accessibility considering solely travel time impedance, ignoring other elements that might hinder access to activities, such as monetary costs (Bocarejo et al., 2014; El-Geneidy et al., 2016; Venter, 2016). These costs are especially relevant to transit-related analyses, since the price of a transit trip might vary according to factors such as route, travel mode and service operator, and therefore is not linearly correlated to travel time (Venter, 2016).

Monetary costs are gradually receiving more attention in the accessibility literature (e.g. Guzman and Oviedo, 2018; Liu and Kwan, 2020; Oviedo et al., 2019). However, there is still little understanding about whether incorporating monetary costs into accessibility measurements can affect the conclusions and policy recommendations derived from transport inequality analyses. Looking at employment accessibility by 
transit in the city of Rio de Janeiro, Brazil, this paper examines how and to what extent simultaneously incorporating travel time and monetary costs into accessibility estimates may impact transport inequality assessments, and how these results compare to those found when following the usual practice of overlooking monetary costs. Rio has recently received growing attention by researchers investigating accessibility and equity issues (e.g. Barboza et al., 2021; Carneiro et al., 2019; Pereira, 2018; Pereira et al., 2019a). Nonetheless, previous examinations have only taken into account travel time impedance, ignoring monetary costs and its constraint effects. In this study accessibility levels were calculated with a cumulative opportunity measure using threshold values both for travel time and monetary cost simultaneously. Analyses were conducted considering different combinations of time and cost thresholds to comprehend how accessibility estimates are affected by the interplay of these variables.

The coming sections are structured as follows: Section 2 presents an overview of recent studies that incorporate monetary costs into accessibility measures; Section 3 exhibits a brief description of Rio's transport system and some of its socioeconomic variables; Section 4 details the data and methods used in the research; Section 5 presents the main results and a discussion on those; finally, Section 6 presents the main conclusions drawn from the results and some recommendations for future studies.

\section{Incorporating monetary costs into accessibility measures}

Incorporating monetary costs into an accessibility measure can increase its sensitivity to the needs and characteristics of individuals (namely their transport-related budget) and to changes in the transport system (since routes and modes can also be described by the costs associated with them), two of the theoretical criteria that accessibility measures should strive to follow, as suggested by Geurs and van Wee (2004). This is especially relevant to location-based measures, widely used for their relative ease of operationalization, but regarded as less theoretically sound than some of their counterparts, like person- and utility-based measures (Geurs and van Wee, 2004).

A few recent studies that use location-based measures have taken the monetary costs into account. Most of these look at how such costs should be considered when 
assessing accessibility and inequalities levels (Bittencourt and Giannotti, 2021; Bocarejo et al., 2014; Bocarejo S. and Oviedo H., 2012; El-Geneidy et al., 2016; Guzman et al., 2017; Guzman and Oviedo, 2018; Liu and Kwan, 2020; Ma et al., 2017; Oviedo et al., 2019; Rodriguez et al., 2017; Van Dijk et al., 2015; Venter, 2016). Others analyze the interaction between accessibility and subjective well-being (Lionjanga and Venter, 2018) and propose a transit path finding method that accounts for monetary constraints (Conway and Stewart, 2019). Most of these studies focus on cities of the Global South, especially in South America and South Africa, perhaps because transport affordability is a more salient issue in the transport equity debate in these contexts than in the Global North. Despite growing attention to monetary costs in accessibility analyses, no previous study has investigated how ignoring monetary cost impedance in accessibility measures, particularly in location-based measures, might systematically skew the results of transport inequality analyses. In other words, no study has yet examined how the usual practice of overlooking monetary costs might render unreal accessibility estimates and, consequently, inequality levels. This paper aims to fill this gap.

Two different methodological approaches are found in the literature when incorporating monetary costs into accessibility measures. Most studies use a generalized cost function, in which a monetary value is assigned to time in order to aggregate time and money into a single total cost. This value of time (VOT) is either set as fixed for the entire study area (e.g. El-Geneidy et al., 2016; Ma et al., 2017) or defined as a function of population-/activities-related attributes that might vary spatially (Oviedo et al., 2019; Venter, 2016).

Generalized cost functions, however, have a few drawbacks. Aggregating time and money into a single value makes interpretation and communication of outcomes more complex. There is no single standard method or recommendation for estimating VOT, which ensues ad-hoc decisions and compromises the comparability of results across different studies. Moreover, there are known limitations about how estimating VOT often raises equity issues (Martens and Di Ciommo, 2017). Some might argue that a fixed VOT produces inaccurate accessibility estimates, as differences in travel behavior and in the perception of VOT across distinct socioeconomic groups are not taken into account. On the other hand, values varying according to individuals’ earnings 
or willingness to pay translate into the contentious notion that wealthier citizens' time is worth more than their poorer counterparts'. In the latter case, inequality assessments could favor historically privileged groups (Goodwin, 1974).

The second approach found in the literature introduces monetary costs into accessibility measures by combining two travel impedance functions, one related to travel times and another to monetary costs. Bittencourt and Giannotti (2021), Conway and Stewart (2019) and Rodriguez et al. (2017) calculate cumulative opportunities measures by simultaneously considering travel time and travel cost thresholds. In this case, the definition of monetary thresholds, likewise for time thresholds, can be done for example according to average spendings or to values deemed acceptable given a budget constraint. This approach is relatively new and it has received less attention in the literature thus far, yet it may be advantageous because it avoids the problem of determining a fair VOT estimate and preserves the easy communication and interpretation of results. For these reasons, we use this approach in the case study described in the following sections.

\section{Study area: Rio de Janeiro}

With more than 6 million inhabitants, Rio de Janeiro is the second most populous city in Brazil and one of the largest cities in Latin America. Though one of the richest cities in the country (IBGE, 2020), it's also one of the most unequal - both in terms of income distribution ${ }^{1}$ and in terms of access to opportunities (Pereira et al., 2019b). The latter is a result of both the spatial distribution of population and employment in the city and the uneven provision of transit infrastructure across its territory.

Population densities in the city tend to increase along a west to east gradient (Figure 1a), with wealthier citizens living in the southern and southeastern regions, as opposed to the poorer citizens, who reside in the northern and northwestern areas

1 In 2010, the Gini of income inequality in Rio was 0.639 and the city ranked as the fourth most unequal Brazilian capital (DATASUS, n.d.). More info at http://tabnet.datasus.gov.br/cgi/idb2011/b09.htm (in portuguese). Income inequality in Rio was comparable to other major cities such as Cape Town, Bogotá or Lagos (Gini between 0.61 and 0.67) and higher than in cities like Santiago, Mexico City, Nairobi and Hong Kong (Gini between 0.53 and 0.55) (UN-HABITAT, 2010). 
(Figure 1b). At the same time, formal jobs concentrate in the CBD, at the eastern end of the city (Figure 1c).
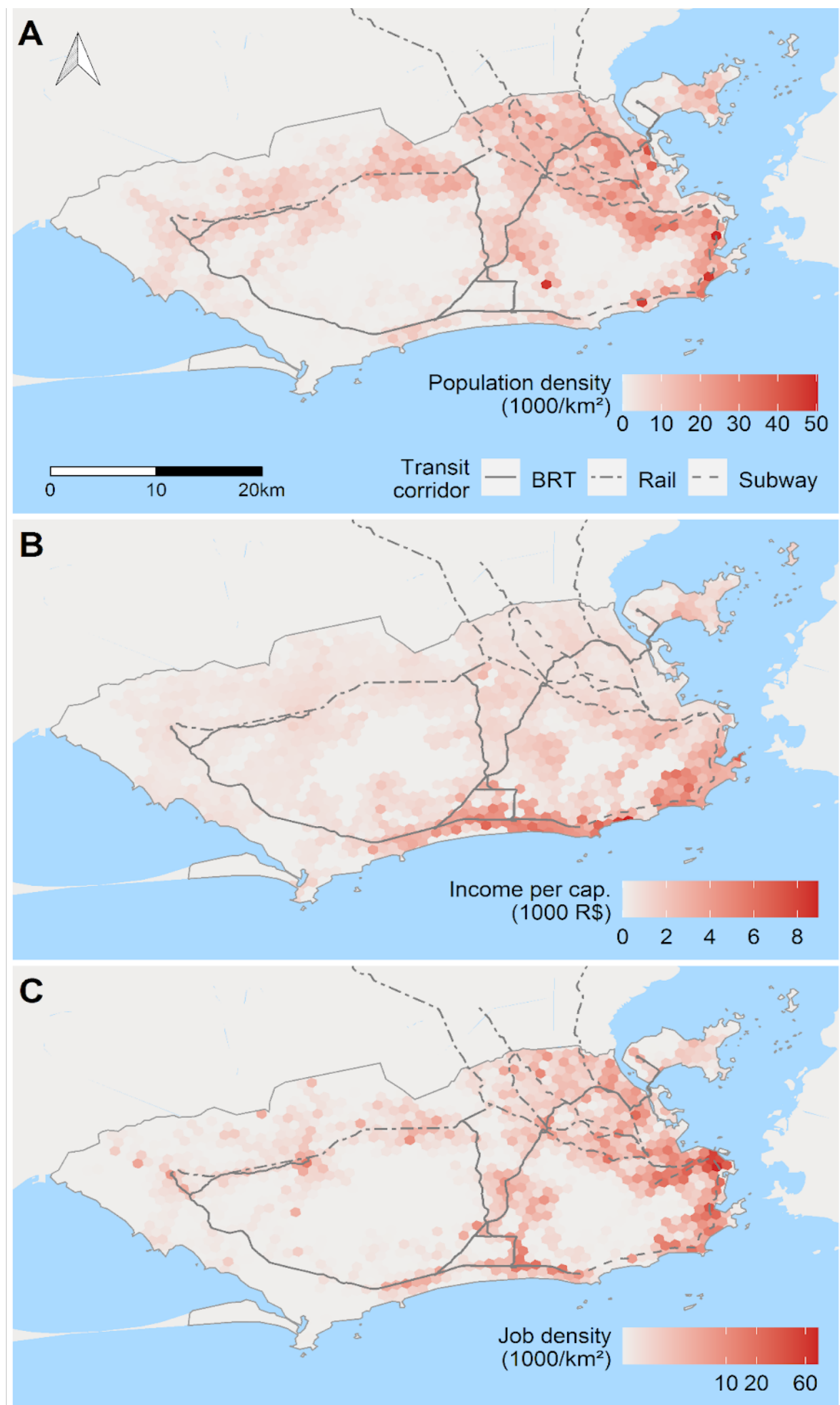
Figure 1: Spatial distribution of population density (A), income per capita (B) and job density (C) in Rio de Janeiro. Population and income data come from the Brazilian Census (2010). Employment data come from the Annual Social Information Relation (2017).

Transit infrastructure in Rio is distributed in a particular way so each mediumand high-capacity transit corridor serves a specific city region, with some overlaps (Figure 2). The subway system runs through the southeastern and the northeastern areas, directly benefiting the upper and middle classes. The rail system runs through the northern and northwestern regions, linking Rio to some of the cities that comprise its Metropolitan Region, and serves the middle and lower classes while also allowing transfers to the subway system at several stations. The BRT system cuts the city diagonally from the southwestern to the northeastern area, which connects to the subway and to the rail system at specific stations. Although reasonably well-distributed, these medium- and high-capacity modes carry only $38 \%$ of the city daily transit passengers: 18\% use the subway, $12 \%$ the rail and 8\% the BRT (Instituto Pereira Passos, 2021).

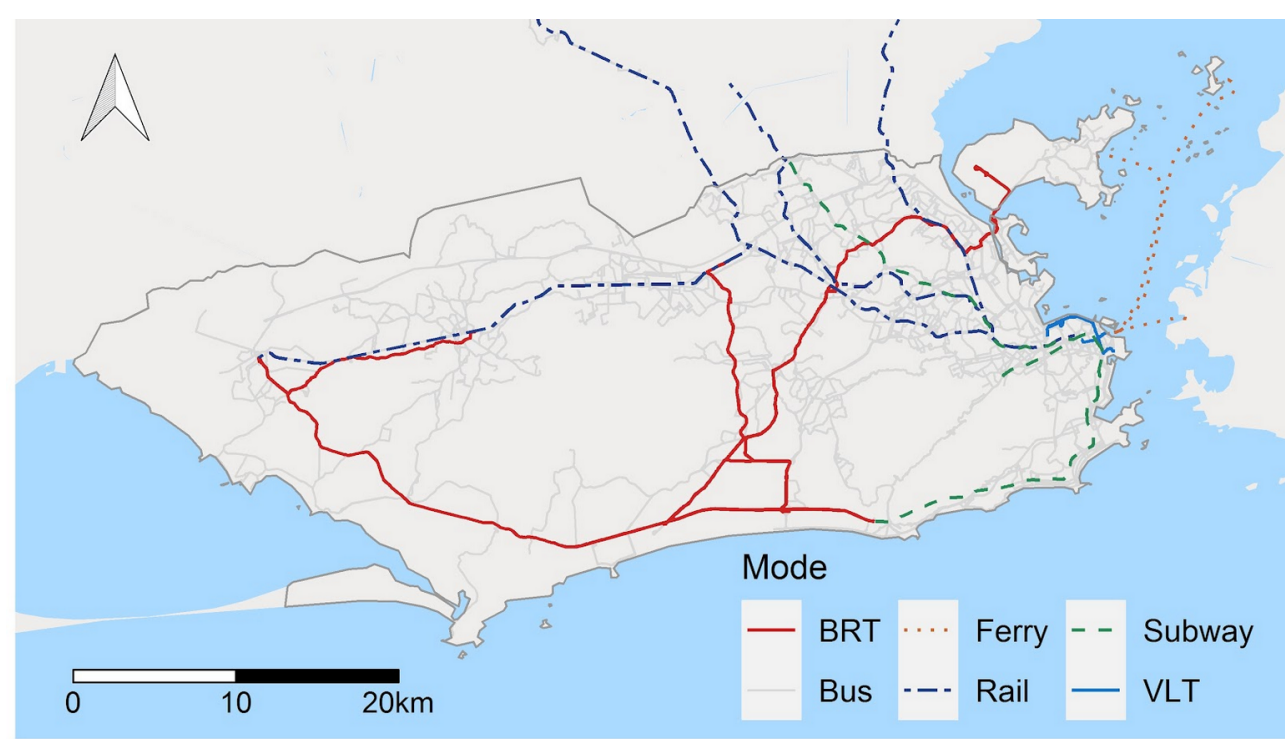

Figure 2: Spatial distribution of Rio’s BRT, municipal bus, rail and subway systems. Transit data come from the Fetranspor and SuperVia GTFS dataset 
The city also counts on a few other transit services: a small light-rail system (VLT) in the city center; a ferry system connecting Rio to the northeastern-most islands and other cities; municipal vans and intermunicipal buses systems, spread throughout the city (not shown on the map); and a widely used municipal bus system.

Bus trips account for $60 \%$ of the daily transit passengers in Rio (Instituto Pereira Passos, 2021). A dense municipal bus network is distributed all around the city, making such buses the only alternative to low-income transit users who live far from the medium- and high-capacity transit corridors. Instead of working in conjunction for a better experience for the users, the buses and the higher capacity modes actually compete for passengers. This is due to the institutional arrangement of the transit system in Rio.

The responsibility for the transit system in Rio lies both with the municipal and state governments. Each entity is responsible for a different set of modes, which they concede the operation and management of to private companies. The municipal government is responsible for the municipal buses, the municipal vans, the VLT and the BRT. The state government, for the subway, the rail, the ferry and the intermunicipal buses. There is, however, little articulation between both government spheres, resulting in a poor experience for the transit user.

Perhaps the main effect of such lack of articulation is the high price paid by the users when making trips that rely on transfers between modes under municipal and state responsibility. Transit users can use the Riocard Mais, a widely available smartcard, to enjoy fare discounts when making two consecutive trips that meet certain criteria. When making transfers between municipal modes, the rule is roughly "two trips by the price of one” (i.e. the second trip is either free of charge or very cheap). Some of the transfers between municipal and state modes and between state modes only, on the other hand, are not covered by the Riocard Mais rules, and those who are require a much larger payment on the second trip than in the municipal modes-only case. Table 1 presents the fare of each mode considered in this paper's analyses, as well as the integration costs between consecutive trips: 
Table 1 - Transit fares in Rio de Janeiro when using the Riocard Mais (values of December 2020) ${ }^{2}$

\begin{tabular}{llll}
\hline \multicolumn{2}{c}{ Single mode } & \multicolumn{2}{c}{ Trip integration } \\
\hline Mode & Fare $\left(\mathbf{R}^{3}\right.$ ) & Integration & Fare (R\$) \\
\hline Light-rail (VLT) & 3.80 & VLT + VLT & 3.80 \\
Municipal bus & 4.05 & Municipal bus + municipal bus & 4.05 \\
BRT & 4.05 & Municipal bus + VLT & 4.05 \\
Rail & 4.70 & Municipal bus + BRT & 4.05 \\
Subway & 5.00 & BRT + Subway & 7.10 \\
Ferry & 6.30 & Rail + Subway & 8.55 \\
& & Bus/BRT + Rail (no discounts) & 8.75 \\
& & Bus + Subway (no discounts) & 9.05 \\
\hline
\end{tabular}

Thus, the buses are not only more widely distributed throughout the city than the higher capacity modes, but they are also cheaper - especially when taking transfers into account. This, when coupled with the fact that several bus routes run along the subway and rail corridors, results in the modal split shown before: many transit users opt for bus trips because, even though they are slower, they cost less. Higher capacity modes, therefore, despite running on some of the poorest regions of the city (especially the rail), are less frequently used than the buses by low-income people as a result of the fare policy.

Combining the spatial configuration, operational characteristics and fare structure of Rio's transit system with the spatial distribution of socioeconomic groups and employment opportunities gives rise to marked transport and accessibility inequalities in the city. Large contingents of low-income neighborhoods located far from the CBD historically face poor employment accessibility conditions (Lago, 2015). Medium- and high-capacity transit corridors could attenuate this issue, but neither they are widely used by the population nor they change the fact that the city's poorest inhabitants have the lowest accessibility levels due to the sheer amount of distance they

2 The values of Table 1 were taken from Riocard Mais' website in December 2020. Please refer to the website for the up-to-date full set of fares: https://www.cartaoriocard.com.br/rcc/institucional/tarifas

3 As of December 2020, R\$ 1000.00 (brazilian reais) was equivalent to approximately USD 192.52. 
have to cover to reach employment centers (Carneiro et al., 2019). Recent transport investments related to the 2014 World Cup and the 2016 Olympic Games were publicized as beneficial to low-income groups, but they have actually increased the accessibility gap between the richest and the poorest (Pereira et al., 2019a).

Previous studies on transport accessibility in the city, however, have overlooked the role of monetary costs in their accessibility estimates (e.g. Bittencourt et al., 2020; Carneiro et al., 2019; Pereira, 2019). This is particularly troubling in Rio, where factors linked to poverty, such as the employment status, are highly correlated with immobility (Motte-Baumvol and Nassi, 2012), and where transit affordability is one of the main topics in the political agenda (ITDP Brasil, 2020). How this fare structure affects accessibility and inequality analyses is a question that remains unanswered, and which we address in the following sections.

\section{Data and methods}

\subsection{Data}

The data used in this paper come from several different sources. Population and socioeconomic data come from the 2010 Brazilian Census, and employment data comes from the 2017 Annual Social Information Relation (RAIS). Both data sets are spatially aggregated and made publicly available by the Access to Opportunities Project (Pereira et al., 2019b). The data were aggregated using a hexagonal grid based on Uber's H3 index (Brodsky, 2018) at resolution 8, where each cell has a 922-meter diagonal, and the city is covered by a total of 1728 hexagons.

Road network and pedestrian infrastructure data were extracted from OpenStreetMap in April 2020. Transit data was provided by Fetranspor and SuperVia in the General Transit Feed Specification (GTFS) format and represent a typical day of operations in November 2018.

Fares were calculated according to the values and rules specified in Riocard Mais (n.d.). Intermunicipal buses were removed from the GTFS datasets, as many of them did not have their fares correct and manual corrections were not possible. These buses are usually more expensive than the other available modes in the city, so removing them 
from the GTFS probably leads to smaller accessibility levels at higher monetary costs thresholds. These routes, however, serve mainly the passengers that travel between Rio and its neighboring cities, not the ones making trips within the city - especially those looking to save money. Since we only look at the accessibility levels within the city of Rio, and focus on the low income population in our policy-related conclusions, this removal does not have significant impacts on our results.

Data collecting, processing and visualization was conducted in R (R Core Team, 2020). The code used in the making of this paper is publicly available on a GitHub repository in order to improve the reproducibility of this study and to promote similar research in different settings ${ }^{4}$.

\subsection{Accessibility estimates}

A cumulative opportunities measure taking both travel time and monetary cost impedance into account was used to estimate accessibility levels throughout the city. This measure has been chosen due to its ease of operationalization, communication and interpretation. It has limitations, however, such as not taking competition effects into account and overlooking individuals' characteristics, like gender and income levels (Geurs and van Wee, 2004; Martens and Golub, 2012). Additionally, cumulative opportunities measures require arbitrarily set cost thresholds, ignoring all activities outside this limit and assuming that all opportunities within it are equally reachable and desirable (Geurs and van Wee, 2004). In order to overcome this issue, various travel time and monetary cost thresholds values were picked from two distinct distributions. Travel time values range from 1 to 90minutes every 1 minute (i.e. $1,2,3 \ldots 88$, 89, 90), while monetary cost values range from $\mathrm{R} \$ 0$ to $\mathrm{R} \$ 15$ every $\mathrm{R} \$ 0.05$ (i.e. R\$ 0, 0.05, $0.10 \ldots 14.90,14.95,15)$. Accessibility levels were then estimated using every possible combination of threshold values from these distributions.

Nonetheless, only a handful of thresholds' combinations are highlighted in the results. We chose to highlight the travel time thresholds of 30, 60 and 90 minutes to demonstrate the impacts of monetary constraints on the accessibility and inequality levels on progressively longer trips. Still, it's worth noting that the average commute time by transit in Rio is 57 minutes and that more than $17 \%$ of transit trips in the city

4 The repository is available at https://github.com/dhersz/msc-thesis. 
were longer than 90 minutes, as per the latest travel survey conducted in the city (Central, 2016).

Monetary costs thresholds, on the other hand, were picked according to Riocard Mais fare rules to emphasize the role that Rio's fare policy and specific modes play on improving the accessibility conditions of different socioeconomic groups:

- $\quad \mathrm{R} \$ 0.00$ - only walking trips;

- $\mathrm{R} \$ 4.05$ - all above and either a single bus, two consecutive buses or consecutive bus and BRT trips;

- $\mathrm{R} \$ 4.70$ - all above and rail trips;

- $\mathrm{R} \$ 5.00$ - all above and subway trips;

- $\quad \mathrm{R} \$ 7.10$ - all above and consecutive brt and subway trips;

- $\mathrm{R} \$ 8.75$ - all above and consecutive bus/BRT and rail trips;

- infinite (no cost limit) - all transport modes available.

While the threshold-combination strategy helps mitigating the boundary effect of the modifiable temporal unit problem (MTUP), related to the arbitrary selection of cost thresholds, our analyses are still susceptible to MTUP’s segmentation and aggregation effects (Pereira, 2019). Temporal aggregation refers to the process of grouping accessibility estimates over a time window and averaging them through a summarizing statistic, usually the median or the mean, to take into account the compound effects of the mismatch between users' departure times and routes' arrival times at transit stops. Temporal segmentation, on the other hand, refers to selecting this time window's starting point.

In this study, departure times were equally distributed every 15 minutes along a 2-hour time window between 7 and 9 am, thus representing a typical weekday during the morning peak. A recent study has shown that cumulative opportunities measures are particularly susceptible to statistical biases related to harmonic error effects, when the selected temporal resolution interacts with the frequency of the transit routes (Stępniak et al., 2019). This study, however, also shows that the outcome of inequality assessments based on estimates at our chosen temporal resolution for the morning peak time window does not vary significantly. Also, this issue is more salient when the transit routes reliably operate on fixed schedules, which is not the case of Rio's transit system. 
Hence, we consider this measure suitable for the goal of demonstrating and communicating the impact of monetary costs on transport inequality analyses in a methodologically robust setting. MTUP’s segmentation effect has not been addressed, since looking at a single time window suffices for the demonstration purposes of this research. Accessibility levels were then calculated as the median number of jobs reachable across such time window, as described in Equations 1 to 3.

$$
\begin{aligned}
& A_{i}=\text { median }_{r \in R}\left(\sum_{j=1}^{n} W_{j} \times \max _{k \in K}\left(f\left(t_{i j r k}\right) \times g\left(c_{i j r k}\right)\right)\right) \\
& f\left(t_{i j r k}\right)=\left\{\begin{array}{l}
1, \text { if } t_{i j k} \leq T \\
0, \text { if } t_{i j k k}>T
\end{array}\right. \\
& g\left(c_{i j r k}\right)=\left\{\begin{array}{l}
1, \text { if } c_{i j r k} \leq C \\
0, \text { if } c_{i j r k}>C
\end{array}\right.
\end{aligned}
$$

where $A_{i}$ is the median transit accessibility to jobs at origin i across all departure times, $R$ is the total set of departure times, $W_{j}$ is the number of jobs at destination $j, n$ is the number of spatial cells covering the study area, $K$ is the total set of itineraries between origin $i$ and destination $j$ at departure time $r, t_{i j k}$ is the travel time of the itinerary $k$ between origin $i$ and destination $j$ at departure time $r, T$ is the travel time threshold, $f\left(t_{i j k}\right)$ is a binary function which takes values 0 or 1 based on the travel time, $c_{i j r k}$ is the monetary cost of the itinerary $k$ between origin $i$ and destination $j$ at departure time $r, C$ is the monetary cost threshold, and $g\left(c_{i j r}\right)$ is a binary function which takes values 0 or 1 based on the monetary cost.

Transit routes were calculated using OpenTripPlanner ${ }^{5}$. Instead of considering only the fastest route between an origin and a destination, which is the usual practice in the literature (e.g. El-Geneidy et al., 2016; Ma et al., 2017), we used OpenTripPlanner to determine up to 20 different transit itineraries for each origin-destination pair, allowing slower but cheaper routes to be considered. Travel times were estimated as the time length of a door-to-door trip, which includes walking from the origin to the departure transit stop, waiting at the stop, in-vehicle time, waiting at occasional transfers and walking from the arrival stop to the destination. Monetary costs were calculated based on the modes and routes that compose each itinerary, assuming that all

5 Available at http://www.opentripplanner.org/. 
city inhabitants are eligible to enjoy the fare discounts made available by the Riocard Mais smartcard (Riocard Mais, n.d.).

In this study we look at how transit fares can affect accessibility estimates without considering how affordable these services are to the population. This is done so as a first exercise to tease out the sole effect of monetary cost thresholds. It is likely that accessibility inequality levels would be higher if transit fares were considered vis-a-vis the population's income distribution, but we leave this analysis to receive careful attention in a future study.

\subsection{Inequality assessment}

In this study we calculate accessibility inequalities using the Palma Ratio. This measure was originally developed by Palma (2011) to investigate income inequalities by dividing the share of national income owned by the richest $10 \%$ by the share owned by the poorest $40 \%$. In the transport equity literature, the measure has been adapted as the ratio between the average accessibility experienced by the richest $10 \%$ population over the experienced by the poorest 40\% population (e.g. Guzman and Oviedo, 2018; Pereira et al., 2019b), as outlined in Equation 4.

$$
P=\frac{\overline{A_{10}}}{\overline{A_{40}}}
$$

where $P$ is the Palma Ratio of the accessibility distribution, $\overline{A_{10}}$ is the richest $10 \%$ 's mean accessibility and $\overline{A_{40}}$ is the poorest $40 \%$ 's mean accessibility.

To calculate the Palma Ratio, we classified each grid cell by income decile considering the size of its population and their average income. Therefore, each decile has the same population size but is composed of a different number of grid cells - while the richest $10 \%$ inhabitants are concentrated on 99 cells, the poorest $40 \%$ inhabitants are distributed among 622 cells across the city.

We have chosen to use the Palma Ratio because of its advantages over other inequality indices, such as the Gini or Theil index. Compared to other measures, the Palma Ratio is easier to communicate and interpret (Guzman and Oviedo, 2018), as it plainly shows how much higher accessibility is for the wealthiest than for the poorest. It also reflects more clearly how accessibility inequality is affected by changes in the 
accessibility levels experienced by the most well-off and the most disadvantaged populations. In contrast, the Gini Index considers how the distribution of accessibility across individuals deviates from hypothetical total equality, but it says nothing about the socioeconomic conditions of those who possess the highest or lowest accessibility levels. A new transport policy that improves the accessibility levels of high-income citizens living in low accessibility areas would reduce overall inequality as captured by the Gini Index, even if no vulnerable populations reaped any benefits from that policy. Hardly this is an equitable outcome.

The Palma Ratio can be questioned for using arbitrary cut-off points (i.e. the richest $10 \%$ and the poorest $40 \%$ ). To address this issue we ran a robustness analysis, recalculating the inequality estimates of this study using the between-groups inequality captured by the Theil Index. These estimates are not presented in the results section for the sake of brevity, but the conclusion of the study holds when using the Theil Index to measure accessibility inequalities.

\section{Results and discussion}

Figure 3 presents the spatial distribution of median job accessibility in the city of Rio de Janeiro when taking into account distinct monetary cost and travel time thresholds. Differences across maps on the same horizontal row highlight the effects of relaxing the travel time threshold on the accessibility levels. Meanwhile, differences across maps on the same vertical column highlight the effects of relaxing the monetary cost threshold on accessibility.

As expected, accessibility levels become higher with higher time and cost thresholds. Looking specifically at the monetary cost limits, one can see that increasing a passengers' transport budget allows her/him to benefit from more options of transit modes, and integrations between them, to enjoy higher accessibility. 
$30 \min$
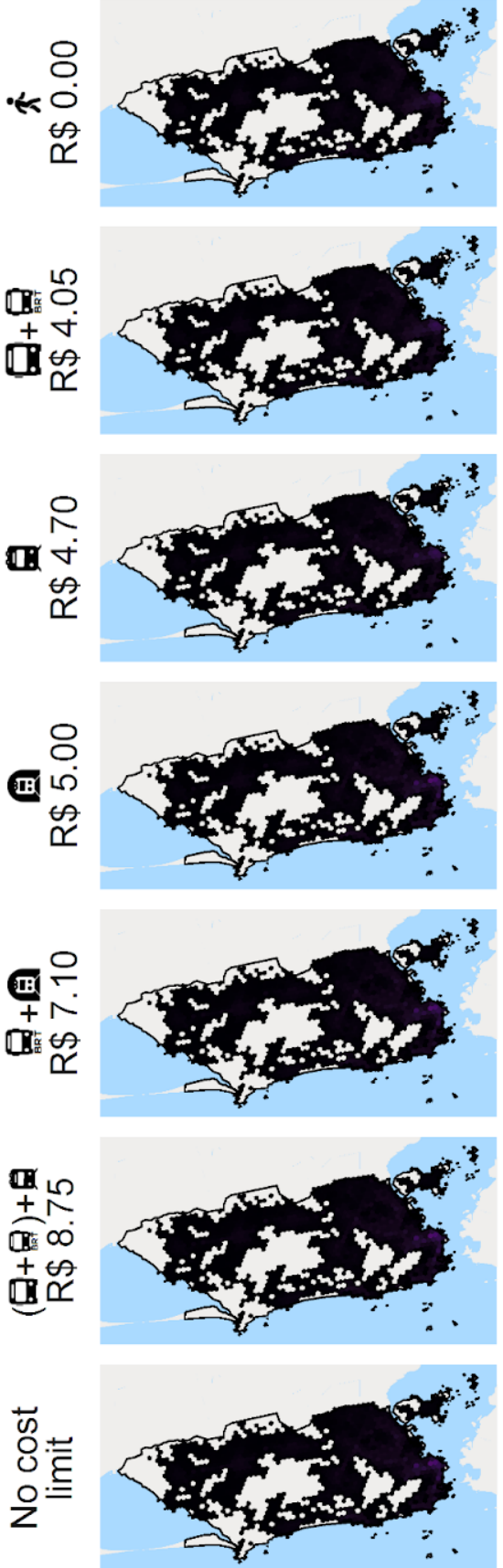

$60 \mathrm{~min}$
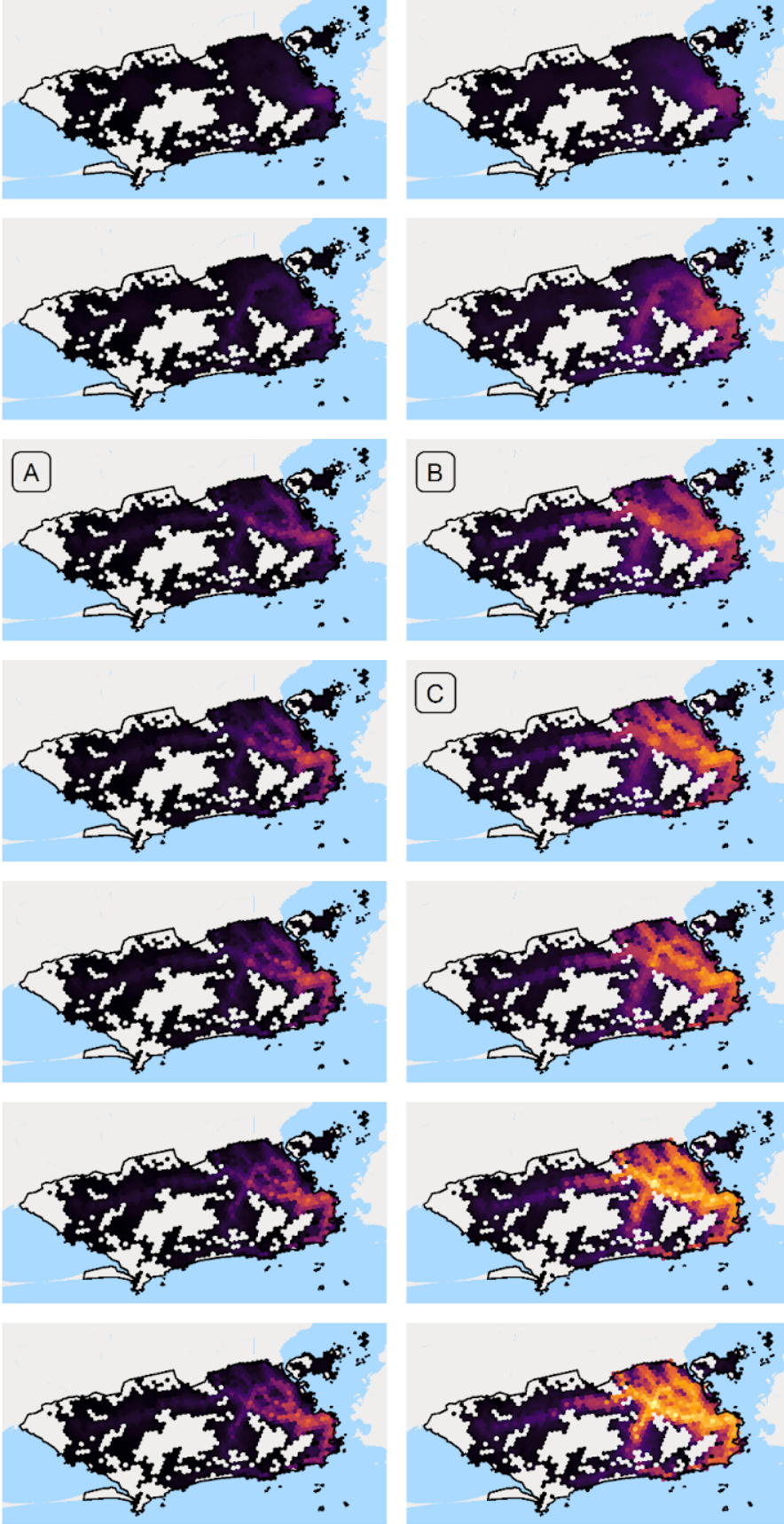

$90 \mathrm{~min}$
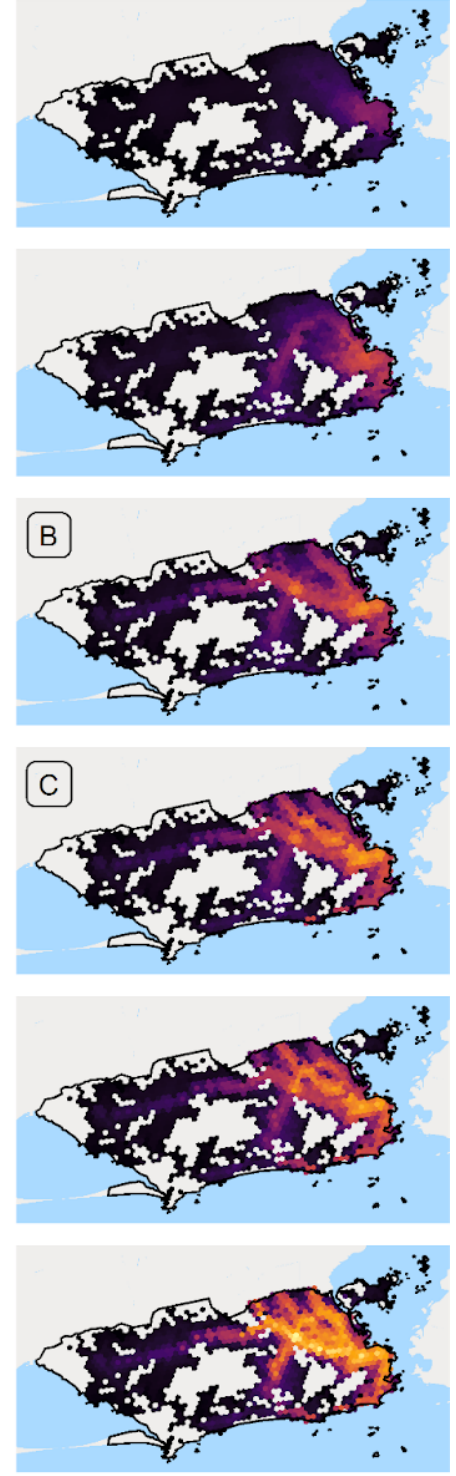

\section{Accessible jobs \\ (\% of total)}

Figure 3 - Spatial distribution of median job accessibility by transit between 7 am and 9 am when taking into account different travel time and monetary cost thresholds. Rio de Janeiro, 2018. 
The accessibility estimates with the $\mathrm{R} \$ 0.00$ threshold reflect the walk accessibility, as there are no free transit modes in the city, which increases in a quasilinear fashion from the CBD outwards. With a R $\$ 4.05$ budget, the user is allowed to make either bus-only (with up to two distinct buses), BRT-only or bus and BRT trips. Bus routes and stops are relatively well distributed across the city, but their low operational speeds hinder large gains in accessibility, particularly in the city’s periphery. The BRT services can deliver larger accessibility gains in part because of its higher speeds, but the BRT network has fewer and more sparse stops compared to regular buses (requiring a greater amount of time spent on access and egress to/from stations), so the accessibility benefits reaped from using this mode only become more apparent over longer time thresholds. Rail routes extending from the CBD to some of the poorest regions of the city come into play at a $\mathrm{R} \$ 4.70$ cost, more perceptibly at higher travel time thresholds due to the large distances that need to be covered to reach the city center. The effects of the subway on accessibility only become noticeable at the $\mathrm{R} \$ 5.00$ threshold. These effects are more pronounced on shorter trips than the aforementioned modes' effects, particularly on the southeastern end of the city, whereas at higher travel time thresholds the accessibility gains occur around the northern extension of the subway corridor.

The impacts of considering transfers between distinct modes are visible at higher monetary cost thresholds. Transit users can enjoy the subway to BRT (and vice-versa) transfer with R \$ 7.10, increasing the accessibility of areas close to these modes' corridors, most notably the BRT surroundings around the geographic center and at the southern end of the city. The combined effects of allowing transfers between the rail (with high operational speed) and regular buses (with higher spatial network connectivity) are shown at the $\mathrm{R} \$ 8.75$ threshold, in which many places throughout the city face accessibility gains, but those are higher around rail stations. Ignoring monetary constraints altogether (the equivalent to setting an infinitely high monetary threshold) leads to even higher accessibility estimates around some transit corridors.

While relaxing either one of the monetary or temporal thresholds leads to higher accessibility levels, each category of threshold affects these levels in different ways. Increasing the travel time thresholds (comparing the maps from left ro right) allows for longer trips, but it does not change the modes that can be used to complete such trips. 
Visually, this results in accessibility hotspots that get gradually larger the higher the travel time, but are still restricted by the "frontiers” of each available mode. Comparing Figures 3A and 3B we see that increasing the travel time cutoff from 60 to 90 minutes while keeping the $\mathrm{R} \$ 4.70$ threshold constant results in higher accessibility around the rail and BRT corridors, but not around the subway (which costs R \$ 5.00). On the other hand, increasing the monetary cost threshold from $\mathrm{R} \$ 4.70$ to $\mathrm{R} \$ 5.00$ while keeping the 90 minutes threshold constant (comparing Figures 3B and 3C) abruptly increases the accessibility levels in the surroundings of the subway corridor, but does not change the accessibility in the rest of the city. This is because relaxing monetary restrictions enables modes that were previously unavailable due to their costs. Visually, this results in the sudden appearance of accessibility hotspots around the corridors of such modes which can be completely unrelated to the previously existing hotspots.

Thus, simultaneously taking both temporal and monetary thresholds into account affects how accessibility is distributed between different socioeconomic groups. Although at first glance the figures seem to indicate that the impact of incorporating monetary costs into accessibility measures is smaller under low travel time thresholds, the effects of such incorporation on the inequality levels suggest otherwise. Figure 4 presents how the magnitude of accessibility inequality between wealthy and poor neighborhoods in Rio vary when considering different travel time and monetary costs constraints.

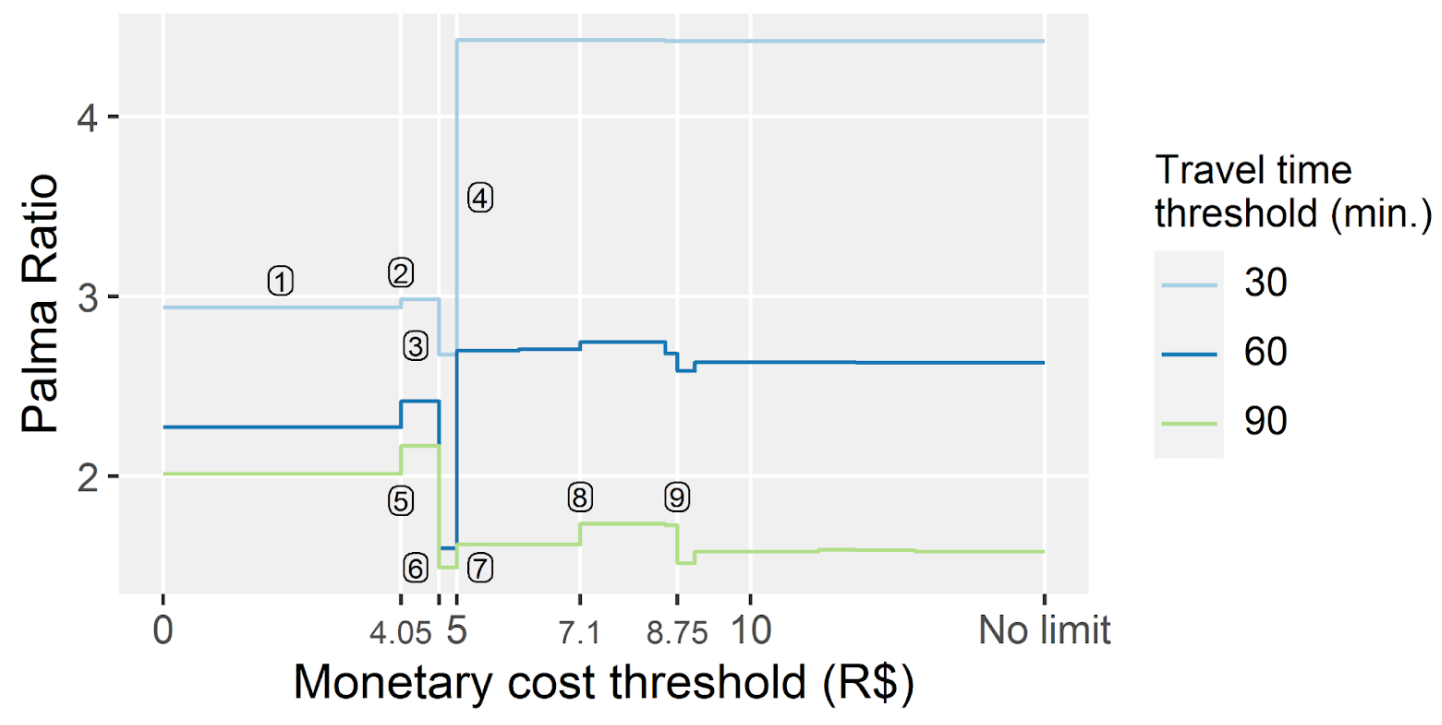


Figure 4 - Palma Ratio variation for each travel time threshold as a result of estimating accessibility with different monetary cost thresholds. Rio de Janeiro, 2018. Obs.: The labeled numbers in the figure indicate data points commented on the text.

The monetary threshold considered in the accessibility estimates strongly impacts the Palma Ratio under every single travel time threshold. Throughout the whole monetary spectrum the inequality tends to be higher at shorter temporal thresholds, as one would expect, because Rio's wealthier neighborhoods tend to be closer to the city's main employment hubs. Curiously, however, with the 30 and 60 minutes travel time thresholds the inequality levels when considering trips more expensive than $\mathrm{R} \$ 5.00$ are higher than when considering walking-only trips, but the same is not true with the 90 minutes threshold. This means that the effect of trip monetary costs on accessibility inequalities can be either positive or negative depending on the temporal threshold considered. A closer look at the figure might help us better comprehend how the interplay between temporal and monetary constraints affect inequality levels.

All curves are flat from $\mathrm{R} \$ 0.00$ to $\mathrm{R} \$ 4.05$ (label 1 in the figure), the value from which the transit system configuration begins to influence inequality levels. At such a value, with which bus and BRT trips become affordable, the 30-minute threshold curve faces a small Palma Ratio increase (2). The distance covered by these modes in such a small amount of time is not enough to substantially increase the number of jobs reachable by the city's poorest inhabitants, but it does boost some of its richest citizens' accessibility levels, as they have to travel smaller distances to reach employment clusters. At R\$ 4.70, when rail trips become affordable, the same temporal threshold curve faces a significant drop (3), only to rise higher than before at $\mathrm{R} \$ 5.00$ (4), when the possibility of using the subway comes into play, and to flatten from this value onwards. Rail corridors run through some of the poorest areas of the city and are completely absent from the richest neighborhoods, therefore contributing to a more equitable transit. The subway, on the other hand, serves some of the city's richest regions, connecting them to the $\mathrm{CBD}$ and other employment hubs. These regions benefit hugely from the subway's high operational speeds, which allow its users to reach many opportunities even when considering travel times as short as 30 minutes. Trips that require a travel budget higher than $\mathrm{R} \$ 5.00$ to be completed inevitably contain a transfer between distinct modes in their composition and can hardly be completed within 30 
minutes due to additional waiting, access and egress time, thus not affecting the inequality levels at this temporal threshold. Though cheaper than buses, the effects of the VLT on inequality levels as measured with the Palma Ratio cannot be seen due to its limited spatial coverage.

The 90-minutes threshold curve pictures a distinct scenario. The Palma Ratio curve also rises at $\mathrm{R} \$ 4.05$ (5) and dips at $\mathrm{R} \$ 4.70$ (6), highlighting the importance of the rail for low-income communities who live very far from the city center. In contrast with the 30-minutes curve, however, the inequality growth at $\mathrm{R} \$ 5.00$ leads to inequality levels lower than those that result from allowing only trips cheaper than R\$ 4.70 (7). This is a sign that the individuals who live in centrally located rich regions gradually "exhaust" the benefits from using the subway alone, so the subway-induced inequality growth decreases with time. Still, considering the trips that include transfers between the subway and the BRT (which cost R\$ 7.10) mainly improves the accessibility conditions of the well-off population who either live next to BRT corridors close to subway stations or can reach many opportunities with short BRT trips after a subway ride (8). The poor population at the far west of Rio, despite being served by the BRT system, live so far from the city center that even a 90-minutes trip may not be enough to reach big employment centers. These individuals take more advantage of trips that include transfers between the municipal buses and the rail, at a R 8.75 fare, which allows for faster access and egress to/from rail stations, consequently increasing their access to many job opportunities and reducing inequality levels as estimated with the Palma Ratio (9). Relaxing monetary constraints beyond R \$ 9.00 stabilizes inequality estimates.

The detailed inspection of the 30- and the 90-minutes threshold curves makes it evident that monetary costs influence the outcomes of transport inequality analyses. No matter the chosen travel time threshold, be it relatively low or quite high, imposing monetary constraints when measuring accessibility significantly changes the observed inequality levels. How these levels are affected is largely influenced by the interplay of monetary and temporal constraints, though. In the case of Rio, for example, taking subway trips into account (at R \$ 5.00) leads to much greater inequality levels increases in the 30-minutes threshold case than in the 90-minutes case. Accounting for rail trips 
(at R \$ 4.70), on the other hand, reduces the inequality to greater extents in the 90minutes threshold case, rather than in the 30-minutes case.

Figure 5 offers a different take on visualizing the effects of monetary and temporal restrictions on accessibility inequality. In contrast to the last figure, it shows how inequality levels within a given monetary threshold fluctuate along different travel time cut-offs. This chart helps us understand the impact of specific modes on inequality levels, and how they change over time, allowing for more thoughtful diagnosis on the role of each transport mode in shaping current accessibility conditions.

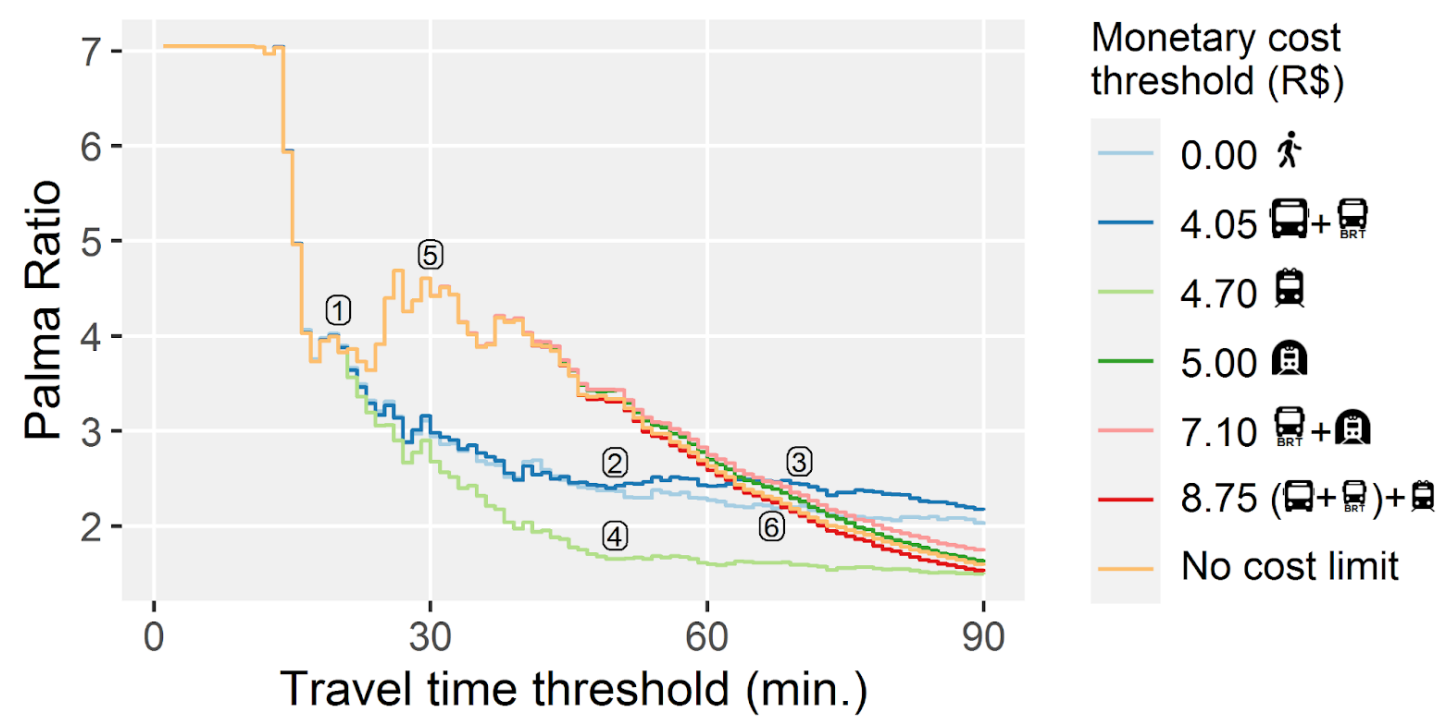

Figure 5 - Palma Ratio variation for each monetary cost threshold as a result of estimating accessibility with different travel time thresholds. Rio de Janeiro, 2018. Obs.: The labeled numbers in the figure indicate data points commented on the text.

The R 0.00 threshold curve represents the baseline scenario, in which no transit service is taken into account and the accessibility inequality is a result of the spatial mismatch between housing and employment. The effects of transit trips on the other curves start to get noticeable around the 20 minutes mark (label 1 in the figure), the point from which it becomes possible to compare the inequality levels that result from taking different combinations of transit modes into account and the baseline scenario.

The inequality when taking municipal bus and BRT trips into consideration, at a cost of R $\$ 4.05$, due to the widespread distribution of bus stops in the city, does not significantly vary from the baseline inequality up to the 50 minutes threshold (2). From 
this point onward the Palma Ratio slightly increases up until around the 70 minutes mark, when it begins to decrease at a faster pace than in the walking-only scenario (3), a sign that only when considering trips as long as at least one hour the poorer population reaps more benefits from these modes than those better-off. Including rail trips in the estimates, contrarily, results in lower inequality levels than in the baseline scenario all the way from the very low to the very high travel time thresholds. The $\mathrm{R} \$ 4.70$ threshold curve steeply declines up to the 50 minutes mark (4), when it starts descending at about the same rate as the walking-only curve. This pattern shows the importance of increasing the fare integration between the rail and other modes in the city, as the accessibility benefits from using this mode alone diminish over time for the poorest inhabitants. All curves from the $\mathrm{R} \$ 5.00$ threshold beyond retain a very similar shape, highlighting the effects of subway trips on inequality levels. Palma Ratio estimates ascend quickly at low travel time limits, but decrease from around the 30 minutes mark onward (5). This goes to show that even though the subway is spatially configured to serve some of the richest inhabitants of the city with short trips to centrally located places, it also benefits poorer individuals at a greater temporal horizon. Still, when compared to the baseline scenario these benefits only outweigh the improved conditions for the rich at around the 70 minutes mark (6).

In summary, these results reinforce the notion that the outcome of inequality assessments not only depends on the monetary threshold set, but on the interplay between monetary and temporal thresholds and, more generally, on the synergy between the spatial configuration of a transit network, the operational attributes of each mode and the fare policy that governs the prices paid by the users. Ultimately, these results emphasize that overlooking monetary constraints when estimating accessibility might lead to inequality levels detached from reality, as they ignore critical characteristics of the transit system. The findings also suggest that, in the specific case of Rio, focusing on improving the access to the rail and lowering the barriers that might prevent a transit user from riding it (specifically addressed here, the monetary cost) is a good path to future policies that seek to mitigate transport inequalities in the city. 


\section{Conclusion}

This study investigated how and to what extent simultaneously incorporating travel time and monetary costs in an accessibility measure impacts the outcomes of transport inequality analyses. The findings show that the common practice of ignoring monetary constraints on accessibility estimates might lead to inequality levels significantly different than those found when taking monetary costs into account.

Not only these costs might exert significant influence over inequality estimates, but the actual interplay between distinct values of monetary and temporal thresholds may critically alter inequality analyses. Comprehending and interpreting the results of such analyses under different combinations of travel time and monetary costs limits requires paying extra attention to how some of the key features of the transit network in the study area (e.g. the spatial configuration of routes and stops, the operational characteristics of each mode and the fare policies that lay down the prices paid for each trip) may shape the accessibility conditions of different population groups.

From a policy point of view, the findings also suggest that in Rio's case the rail is a vital mode to combat transport inequalities, and therefore should be prioritized in future transport policies and investments. Given the transit infrastructure that is currently in place in the city, the rail corridors extend the furthest into some of the city's poorest neighborhoods and offer high operational speeds and capacity, allowing poor inhabitants to reach large employment hubs. The results show that changes in the fare policy alone could potentially increase the accessibility of many low-income individuals if they reduced or eliminated additional costs imposed by transfers to the rail corridors (e.g. by offering fare discounts on transfers between the municipal buses and the rail, which are currently unsubsidized). A fare integration policy of this kind could probably be cheaper than building new BRT or subway infrastructure, for example.

The analyses conducted in this paper show that conclusions and policy recommendations derived from transport inequality analyses can be affected in nonintuitive ways by the interplay between travel time and monetary costs constraints. The results presented here, however, cannot be easily generalized to other contexts, as they are largely a result of the complex interaction between the spatial organization, operational characteristics and fare policy of Rio’s transit systems and the spatial 
mismatch between jobs and residential locations in the city. Nevertheless, this study highlights the importance of taking local transit system's characteristics into account when analyzing the effects of monetary costs on accessibility inequalities. Further research is necessary to understand how and to what extent different fare policies and combinations of temporal and monetary thresholds impact the result of transport inequality analyses conducted in different contexts.

It's important to note that not addressing monetary costs explicitly (i.e. not setting a price limit for trips, for example) should not be seen as abstaining from taking the effects of monetary costs on accessibility estimates into account. Instead, it means conducting an accessibility analysis based on the unrealistic assumption that all individuals equally face no transport budget constraints. This assumption has been shown problematic in the case of Rio, in which inequality levels significantly vary between the no cost limit and some of the more cost-restrictive scenarios. Explicitly incorporating monetary restrictions into our accessibility measure resulted in a more accurate and correct diagnosis of transport-related inequalities in the city. This diagnostic would be incomplete, at best, and could be potentially misleading, at worst, had we only considered travel time restrictions. While we do believe that monetary costs play an important role in shaping the transport inequalities worldwide, further research is necessary to understand the extent to which not addressing such costs explicitly might skew the results of inequalities analyses and subsequent policy recommendations in different contexts.

This study looked at the plain monetary costs of transit trips, overlooking how affordable these services are to the population. Transit affordability is a central topic to transport justice that plays a very decisive role in the forging of accessibility and mobility conditions of poor individuals across the globe. While our results show that even plain costs can have a big impact on the outcome of inequality appraisals, taking affordability into account may very well increase the accessibility gap between the richest and the poorest even further, so future studies should aim to include this element in their estimates and analyses.

Another limitation of this study is that it only looks at the cumulative opportunities measure. Although a good indicator that monetary costs might affect all types of accessibility estimates, due to the very nature of distinct spatially distributed 
transit services being offered at different prices and service levels, the thresholds-based analyses conducted here are hardly translatable to other widely used accessibility measures, such as competitive and potential measures. Further investigation is therefore required to understand how inequality assessments that make use of such measures may change as a result of incorporating monetary costs impedance.

\section{Acknowledgements}

The authors thank Fetranspor and Supervia for providing the Rio de Janeiro’s GTFS datasets upon request via e-mail. Funding: this work was supported by the Conselho Nacional de Desenvolvimento Científico e Tecnológico (CNPq) and the Instituto de Pesquisa Econômica Aplicada (IPEA).

\section{References}

Barboza, M.H.C., Carneiro, M.S., Falavigna, C., Luz, G., Orrico, R., 2021. Balancing time: Using a new accessibility measure in Rio de Janeiro. J. Transp. Geogr. 90, 102924. https://doi.org/10.1016/j.jtrangeo.2020.102924

Bittencourt, T.A., Giannotti, M., 2021. The unequal impacts of time, cost and transfer accessibility on cities, classes and races. Cities 116, 103257. https://doi.org/10.1016/j.cities.2021.103257

Bittencourt, T.A., Giannotti, M., Marques, E., 2020. Cumulative (and selfreinforcing) spatial inequalities: Interactions between accessibility and segregation in four Brazilian metropolises. Environ. Plan. B Urban Anal. City Sci. 239980832095842. https://doi.org/10.1177/2399808320958426

Bocarejo, J.P., Portilla, I.J., Velásquez, J.M., Cruz, M.N., Peña, A., Oviedo, D.R., 2014. An innovative transit system and its impact on low income users: the case of the Metrocable in Medellín. J. Transp. Geogr. 39, 49-61. https://doi.org/10.1016/j.jtrangeo.2014.06.018

Bocarejo S., J.P., Oviedo H., D.R., 2012. Transport accessibility and social inequities: a tool for identification of mobility needs and evaluation of transport investments. J. Transp. Geogr. 24, 142-154. https://doi.org/10.1016/j.jtrangeo.2011.12.004

Brodsky, I., 2018. H3: Uber’s Hexagonal Hierarchical Spatial Index [WWW Document]. Uber Eng. Blog. URL https://eng.uber.com/h3/ (accessed 4.22.21). 
Carneiro, M., Toledo, J., Aurélio, M., Orrico, R., 2019. Espraiamento urbano e exclusão social. Uma análise da acessibilidade dos moradores da cidade do Rio de Janeiro ao mercado de trabalho. EURE Santiago 45, 51-70. https://doi.org/10.4067/S0250-71612019000300051

Central, 2016. Relatório 4 - Planejamento e Execução das Pesquisas: Parte 3: Diagnóstico da Situação Atual. Companhia estadual de transportes e logística (Central), Governo do Estado do Rio de Janeiro, Rio de Janeiro.

Conway, M.W., Stewart, A.F., 2019. Getting Charlie off the MTA: a multiobjective optimization method to account for cost constraints in public transit accessibility metrics. Int. J. Geogr. Inf. Sci. 33, 1759-1787. https://doi.org/10.1080/13658816.2019.1605075

DATASUS, n.d. Índice de Gini da renda domiciliar per capita - Brasil [WWW Document]. URL http://tabnet.datasus.gov.br/cgi/ibge/censo/cnv/ginibr.def (accessed 6.2.21).

El-Geneidy, A., Levinson, D., Diab, E., Boisjoly, G., Verbich, D., Loong, C., 2016. The cost of equity: Assessing transit accessibility and social disparity using total travel cost. Transp. Res. Part Policy Pract. 91, 302-316. https://doi.org/10.1016/j.tra.2016.07.003

Geurs, K.T., van Wee, B., 2004. Accessibility evaluation of land-use and transport strategies: review and research directions. J. Transp. Geogr. 12, 127-140. https://doi.org/10.1016/j.jtrangeo.2003.10.005

Goodwin, P.B., 1974. Generalised time and the problem of equity in transport studies. Transportation 3, 1-23. https://doi.org/10.1007/BF02351839

Guzman, L.A., Oviedo, D., 2018. Accessibility, affordability and equity: Assessing 'pro-poor' public transport subsidies in Bogotá. Transp. Policy 68, 37-51. https://doi.org/10.1016/j.tranpol.2018.04.012

Guzman, L.A., Oviedo, D., Rivera, C., 2017. Assessing equity in transport accessibility to work and study: The Bogotá region. J. Transp. Geogr. 58, 236-246. https://doi.org/10.1016/j.jtrangeo.2016.12.016

IBGE, 2020. Produto interno bruto dos municípios 2018, Contas Nacionais. IBGE, Rio de Janeiro.

Instituto Pereira Passos, 2021. data.rio: Informações sobre a cidade - Tabelas 728, 1736, 3603 [WWW Document]. data.rio. URL https://www.data.rio/ (accessed 6.28.21).

ITDP Brasil, 2020. O carioca e o transporte na cidade.

Lago, L.C. do, 2015. Desigualdades e segregação na metrópole: o Rio de Janeiro em tempo de crise, 2nd ed. Letra Capital, Rio de Janeiro. 
Lionjanga, N., Venter, C., 2018. Does public transport accessibility enhance subjective well-being? A study of the City of Johannesburg. Res. Transp. Econ. 69, 523535. https://doi.org/10.1016/j.retrec.2018.07.011

Liu, D., Kwan, M., 2020. Measuring Job Accessibility Through Integrating Travel Time, Transit Fare And Income: A Study Of The Chicago Metropolitan Area. Tijdschr. Voor Econ. En Soc. Geogr. 111, 671-685. https://doi.org/10.1111/tesg.12415

Lucas, K., van Wee, B., Maat, K., 2016. A method to evaluate equitable accessibility: combining ethical theories and accessibility-based approaches. Transportation 43, 473-490. https://doi.org/10.1007/s11116-015-9585-2

Ma, Z. (Eric), Masoud, A.R., Idris, A.O., 2017. Modeling the Impact of Transit Fare Change on Passengers’ Accessibility. Transp. Res. Rec. J. Transp. Res. Board 2652, 78-86. https://doi.org/10.3141/2652-09

Martens, K., 2012. Justice in transport as justice in accessibility: applying Walzer's 'Spheres of Justice' to the transport sector. Transportation 39, 1035-1053. https://doi.org/10.1007/s11116-012-9388-7

Martens, K., Di Ciommo, F., 2017. Travel time savings, accessibility gains and equity effects in cost-benefit analysis. Transp. Rev. 37, 152-169. https://doi.org/10.1080/01441647.2016.1276642

Martens, K., Golub, A., 2012. A justice-theoretic exploration of accessibility measures, in: Accessibility Analysis and Transport Planning. Edward Elgar Publishing, pp. 195-210. https://doi.org/10.4337/9781781000113.00020

Motte-Baumvol, B., Nassi, C.D., 2012. Immobility in Rio de Janeiro, beyond poverty. J. Transp. Geogr. 24, 67-76. https://doi.org/10.1016/j.jtrangeo.2012.06.012

Oviedo, D., Scholl, L., Innao, M., Pedraza, L., 2019. Do Bus Rapid Transit Systems Improve Accessibility to Job Opportunities for the Poor? The Case of Lima, Peru. Sustainability 11, 2795. https://doi.org/10.3390/su11102795

Palma, J.G., 2011. Homogeneous Middles vs. Heterogeneous Tails, and the End of the 'Inverted-U': It's All About the Share of the Rich. Dev. Change 42, 87-153. https://doi.org/10.1111/j.1467-7660.2011.01694.x

Pereira, R.H.M., 2019. Future accessibility impacts of transport policy scenarios: Equity and sensitivity to travel time thresholds for Bus Rapid Transit expansion in Rio de Janeiro. J. Transp. Geogr. 74, 321-332. https://doi.org/10.1016/j.jtrangeo.2018.12.005

Pereira, R.H.M., 2018. Transport legacy of mega-events and the redistribution of accessibility to urban destinations. Cities 81, 45-60. https://doi.org/10.1016/j.cities.2018.03.013

Pereira, R.H.M., Banister, D., Schwanen, T., Wessel, N., 2019a. Distributional effects of transport policies on inequalities in access to opportunities in Rio de Janeiro. J. Transp. Land Use 12. https://doi.org/10.5198/jtlu.2019.1523 
Pereira, R.H.M., Braga, C.K.V., Serra, B., Nadalin, V.G., 2019b. Desigualdades socioespaciais de acesso a oportunidades nas cidades brasileiras, 2019. Texto Para Discussão IPEA 2535, 58.

Pereira, R.H.M., Schwanen, T., Banister, D., 2017. Distributive justice and equity in transportation. Transp. Rev. 37, 170-191. https://doi.org/10.1080/01441647.2016.1257660

R Core Team, 2020. R: A Language and Environment for Statistical Computing. R Foundation for Statistical Computing, Vienna, Austria.

Riocard Mais, n.d. Tarifa [WWW Document]. URL https://www.cartaoriocard.com.br/rcc/institucional/tarifas (accessed 12.22.20).

Rodriguez, C., Peralta-Quirós, T., Guzman, L.A., Cárdenas Reyes, S.A., 2017. Accessibility, Affordability, and Addressing Informal Services in Bus Reform: Lessons from Bogotá, Colombia. Transp. Res. Rec. J. Transp. Res. Board 2634, 35-42. https://doi.org/10.3141/2634-06

Stępniak, M., Pritchard, J.P., Geurs, K.T., Goliszek, S., 2019. The impact of temporal resolution on public transport accessibility measurement: Review and case study in Poland. J. Transp. Geogr. 75, 8-24. https://doi.org/10.1016/j.jtrangeo.2019.01.007

UN-HABITAT, 2010. State of the World's Cities 2010/2011- Cities for All: Bridging the Urban Divide. UN-HABITAT.

Van Dijk, J.T., Krygsman, S., De Jong, T., 2015. Toward spatial justice: The spatial equity effects of a toll road in Cape Town, South Africa. J. Transp. Land Use. https://doi.org/10.5198/jtlu.2015.555

Venter, C., 2016. Assessing the potential of bus rapid transit-led network restructuring for enhancing affordable access to employment - The case of Johannesburg's Corridors of Freedom. Res. Transp. Econ. 59, 441-449. https://doi.org/10.1016/j.retrec.2016.05.006 